\title{
An improved method for pylon extraction and vegetation encroachment analysis in high voltage transmission lines using LiDAR data
}

\author{
Nosheen Munir \\ School of Info and Comm Tech \\ Griffith University, QLD 4111 \\ Australia \\ nosheen.munir@griffithuni.edu.au
}

\author{
Mohammad Awrangjeb \\ School of Info and Comm Tech \\ Griffith University, QLD 4111 \\ Australia \\ m.awrangjeb@griffith.edu.au
}

\author{
Bela Stantic \\ School of Info and Comm Tech \\ Griffith University, QLD 4222 \\ Australia \\ b.stantic@griffith.edu.au
}

\begin{abstract}
The maintenance of high-voltage power lines rightsof-way due to vegetation intrusions is important for electric power distribution companies for safe and secure delivery of electricity. However, the monitoring becomes more challenging if power line corridor (PLC) exists in complex environment such as mountainous terrains or forests. To overcome these challenges, this paper aims to provide an automated method for extraction of individual pylons and monitoring of vegetation near the PLC in hilly terrain. The proposed method starts off by dividing the large dataset into small manageable datasets. A voxel grid is formed on each dataset to separate power lines from pylons and vegetation. The power line points are converted into a binary image to get the individual spans. These span points are used to find nearby vegetation and pylon points and individual pylons and vegetation are further separated using a statistical analysis. Finally, the height and location of extracted vegetation with reference to power lines are estimated and separated into danger and clearance zones. The experiment on two large Australian datasets shows that the proposed method provides high completeness and correctness of $96.5 \%$ and $99 \%$ for pylons, respectively. Moreover, the growing vegetation beneath and around the PLC that can harm the power lines is identified.
\end{abstract}

Index Terms-Power lines, pylons, vegetation, span, power line corridor

\section{INTRODUCTION}

Electricity has become one of the indispensable form of energy and power line plays a vital role in the normal manoeuvre of modern societies [1]. A power line system is made up of interconnecting networks such as power plant, transmission lines and electric pylons. The safe delivery of electricity to the consumers is the first and foremost duty of electric power companies nowadays. The over grown trees near the power line corridor (PLC) is one of the potential risks and needs to be monitored regularly. For instance, growing vegetation underneath the transmission line and wind-blown trees are possible threats [2], which can cause widespread power failures, bushfires and have significant impact on financial cost [3].

The monitoring of PLC primarily includes two of the following aspects: electrical components such as power lines and pylons and surrounding objects, such as vegetation. For reliable transmission the stability of wires and pylons and monitoring of vegetation near PLC is important [1]. Furthermore, the highvoltage power lines spread over intricate environments (e.g., forest, mountains) and their distribution has become more complicated [4], which makes power line inspection more challenging.

With an improvement of remote sensing technologies and innovative data processing algorithms, various unconventional remote sensing power platforms such as optical image [5]; SAR (synthetic aperture radars) [6]; thermal image [7]; and LiDAR (Light detection and ranging) [1], [8]; have been used for supervision of smart networks. Compared with other remote sensing technologies LiDAR does not have limitations such as weather dependency and shadow and can collect highdensity three dimensional (3D) point clouds accurately over large areas, and has emerged as a powerful tool for efficient inspection in recent years.

Monitoring of vegetation around power lines is generally a less common research area than extraction of power line components [1]. In spite of its importance and demands to develop new approaches, there have been not much research efforts that are directly related to power line scene monitoring and modelling using airborne LiDAR data. Also, the extraction of individual pylons, power lines and trees or vegetation is very important for modelling of PLC objects and monitoring of vegetation around the PLC.

This paper presents an automated approach for extraction of pylon and vegetation as well as later using vegetation location and height with reference to nearby power lines to express potential hazards. To handle the presence of hilly terrain and near PLC, the dataset is firstly divided into small datasets from the input point cloud data and a voxel grid is created on each dataset to separate pylons, vegetation and power lines. The power line points from the previous step are converted into a binary image to detect the spans in a given segment. Pylons and vegetation located close to each span are extracted and pylons are further separated from vegetation. Finally, the extracted trees and vegetation near the span are considered and their height with reference to minimum power line height is 
estimated to divide them into clearance and danger zones. The remaining paper is designed as follows. The related work is presented in Section II and Section III introduces a work flow of the proposed method. The parameters which are set to achieve the proposed task are outlined in Section IV. The performance study that includes datasets, the ground truth and evaluation metrics followed by results is presented in Section V. Finally, conclusions drawn from experiments and test are discussed in section VI.

\section{PREVIOUS WORKS}

This section briefly describes the existing methods for pylon detection, pylon extraction and vegetation monitoring for PLC.

\section{A. Pylon Detection and Extraction}

In the literature, the pylon extraction methods mainly fall into two categories: supervised and unsupervised classification methods. The supervised methods used supervised classifiers for extraction of pylon points. For instance Kim and Sohn computed 21 features using voxel and sphere based neighbourhoods and used the Random Forest (RF) classifier to categorise data into five classes: ground, vegetation, pylons, power lines and buildings [9]. Guo et al. proposed a contextual JointBoost-based classifier method to classify airborne point cloud data into five categories using different geometric and echo features [10]. However, these methods require large training datasets which are hard to collect for desired results and an unbalanced sampling in the training set increases the rate of misclassification.

The most of the unsupervised methods use statistical analysis i.e., image based methods or pattern recognition techniques to detect and extract pylons. Sohn et al. [11] used the RANSAC (Random SAmple Consensus) algorithm to extract 3D lines. Then, these lines were transformed into a binary image and the RF classifier was used to classify 2D lines by using their orientation properties. This method used supervised and unsupervised methods for pylon detection and did not perform the individual pylon extraction. Recently, Awrangjeb et al. [12] first generated a power line mask to get successive pylons linked to each other through power lines. Then, a pylon mask was generated without power lines. A combined connected component and histogram analysis was performed to find the individual pylons. In another work of Awrangjeb [13] the PLC was first extracted in the form of rectangular regions and pylon locations were detected by finding the gap in terms of height between vegetation and power lines. The pylon locations were used as seed regions and grown to extract the complete pylons. However, if vegetation in the PLC reached the power line height in the middle or anywhere in the span, the estimation of heights gaps could give wrong results.

\section{B. Vegetation Monitoring}

In general, forest monitoring from airborne laser scanner (ALS) data is a topic of active research [1] and transmis- sion line (TL) inspection has drawn a lot of attention as an important application of LiDAR technology. But most of the studies in literature used aerial video surveillance [14], aerial multispectral imaging [15] and mobile laser scanner (MLS) [16] to find vegetation that can harm the power lines. Recently, Shi et al. [17] provided the potential hazard analysis of vegetation near TL using MLS. However, they used Digital Forestry toolbox to extract individual trees. Also its application is only feasible to urban areas due to limitations of MLS technology in forest area.

Also, there are many methods available for individual tree recognition from point cloud data and attaining the structure information of trees [18]. Some studies have combined aerial images and ALS data in vegetation monitoring in PLC [19]. However, there is no work that uses vegetation information to monitor PLC through the ALS data. To our knowledge, no studies exist that have used the ALS data in the mapping of vegetation in the complex environment of a PLC.

\section{APPROACH AND METHODOlOGY}

The proposed method used unsupervised processing techniques that incorporates both images based and point based methods and does not employ any machine learning technique. As shown in Figure 1 the proposed method is based on three steps: span detection and extraction, pylon and vegetation extraction, and vegetation potential hazard analysis. In the span detection and extraction step, the dataset is divided into small segments and a $3 \mathrm{D}$ voxel grid is formed on each segment to separate power lines from pylons and vegetation by using the fact that pylons and trees are vertical while power lines are non-vertical objects. The non-vertical points in the previous step represent power line points which are used to form a binary mask. The binary image is flood filled using the span width threshold which helps to detect individual spans in a given segment. In pylons and vegetation extraction step, the span points are used to find close vertical points that include the vegetation beneath and around the span and pylon. The statistical analysis is adopted on the nearby vertical points to separate pylons from vegetation by exploiting the fact that pylons reach the maximum power line height. The individual trees are extracted and vegetation which is in the form of shrubs is split into small pieces. Finally, in the last step, the extracted trees, bushes and shrubs in a span proximity are considered and their height from the minimum power line point is calculated to categorise them into clearance and danger zones.

\section{A. Span Detection and Extraction}

Figure 2 shows a sample data that will be used to demonstrate the steps for span detection and extraction. Firstly, height of $1 \mathrm{~m} \mathrm{[12]}$ is added to the local digital terrain model (DTM) height to separate the non-ground points from the rest, which contains the ground points and low height vegetation.

Although the DTM can be generated from the LiDAR data, it is assumed it is available with the input data. If not available, it can be generated by using software like MARS Explorer. The 


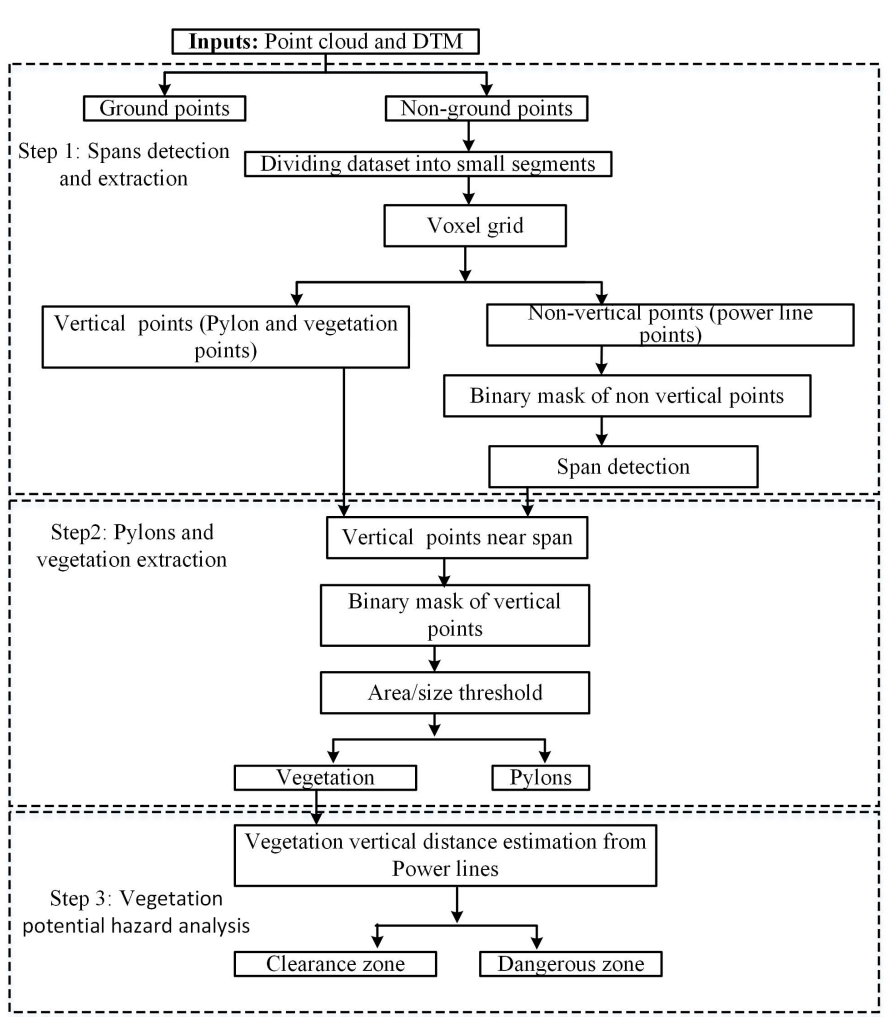

Fig. 1. Flow chart of the proposed methodology.

non-ground points, as shown in Figure 2(c), are expected to contain only the objects of interest, such as pylons and power lines and trees in this case. It provides an approximate ground height for each point in the LIDAR data. Thus, the height values are estimated as relative heights from the ground in the rest of the paper.

1) Estimating Segments in Dataset: Airborne LiDAR systems usually acquire a large number of non-interest points because of the flying height and the scanning angle of the aircraft. The input point cloud data may contain millions of 3D points in any given $1 \mathrm{~km}^{2}$ scene and have points from both natural and structural objects. The objective is to find the vegetation near the TL within a certain distance which can be a real threat for the safety of power lines.

To reduce the number of point clouds for subsequent processing and to process it efficiently, the large dataset is split into smaller and more manageable datasets. The number of observations in each smaller sized dataset will be equal to a given number except possibly for one smaller sized dataset, this might have smaller number of observations than the given number. To perform this job, a simple data step technique is employed to divide the large dataset into smaller datasets. Each sub dataset or segment contain roughly 10 million points.

The size and number of segments can be increased or decreased depending upon the size of dataset and resources such as computer power etc. In addition to that, to increase the efficiency, parallel computing can be employed to process

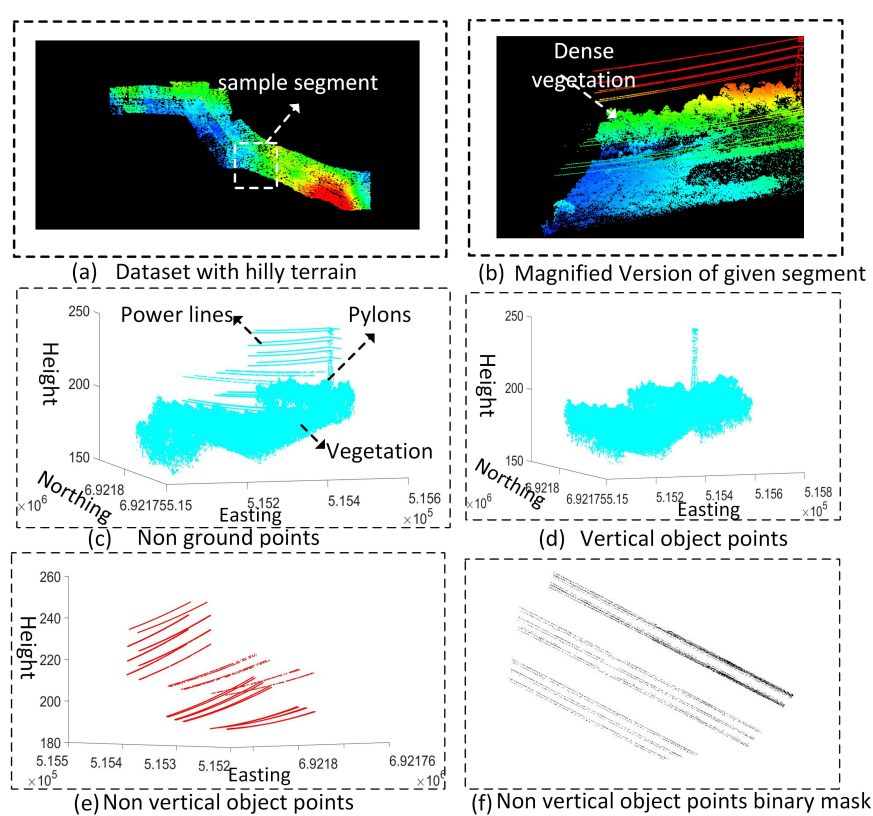

Fig. 2. Span detection and extraction in the sample scene.

each dataset for further processing to extract pylons, power lines and vegetation simultaneously as compare to alternative methods proposed in a vegetated area (e.g., forest) that worked on the whole dataset simultaneously.

2) Extracting Vertical and Non-vertical Object Points: The $3 \mathrm{D}$ voxel grid of size $5 \mathrm{~m} \times 5 \mathrm{~m} \times 5 \mathrm{~m}$ are generated over each dataset from the previous step. The 3D grid makes the processing complicated but unlike the $2 \mathrm{D}$ grid it does not compromise on geometrical information of points in each grid cell (voxel). The size of voxel should be selected wisely while too small make the processing more complex and too big will not provide the effective result.

To separate the vertical and non-vertical points, each cuboid in a grid is further divided into fixed sections of $1 \mathrm{~m}$ height and the vertical and non-vertical property of pylons, trees and power lines is exploited to divide these objects into vertical and non-vertical point categories [9]. The number of "continuous on segments" $C_{n}$, i.e., the consecutive segments with points, and the number of "continuous off segments" $C_{f}$, i.e., the consecutive segments with no points, for each voxel are calculated. While power lines show low value for $C_{n}$ but high value for $C_{f}$, vegetation and pylons displays the opposite. Figures 2 (d) and (e) show the vertical and non-vertical points from the given segment.

3) Spans Detection in each Segment: The non-vertical points as shown in Figure 2 (e) that contain only the power line points from the previous step is transformed into a binary mask $M$ using the method described in [12]. The resolution $R_{M}$ of $M$ is set fixed at 0.25 and all pixels are initially filled with 1 (white). Then, for each non-ground point within a pixel, a neighbourhood (e.g., $3 \times 3$, consistent with the point density) is filled with 0 (black). The value of $R_{M}$ is critical here, as 
the input point cloud data can be sparse and the value of $R_{M}$ should not be large that will make the mask tiny in size and power lines become too small and makes the mask mystifying. Figure 2 (f) displays the binary mask $M$ with only wire points (marked with 0, black). It is noticeable in Figure 2 (f), the power lines that belong to the same span are much closer as compared to those that belong to a different span. Also, power lines in the same span are close to one another but not connected, thus $M$ is flood filled with black to remove white holes to connect the power line points that belong to the same span. Assuming the maximum dimension of a span width is $W_{d}=20 \mathrm{~m}$, power line points in the same span are flood filled with this threshold.

After the neighbourhood filling, the individual span points are found connected in the final mask as shown in Figure 3 (a). Lastly, a connected component analysis (CCA) is carried out on the filled image to obtain individual span boundaries.

\section{B. Pylons and Vegetation Extraction}

For each connected component (CC), the span points within its boundary are converted back to $3 \mathrm{D}$ points. The mean of span points is estimated and the vertical points which are close to mean of span points are extracted by setting a distance threshold $T_{d}$ of $30 \mathrm{~m}$. So, the points with in $30 \mathrm{~m}$ location are assumes that they are close to the corridor in which span is located. By doing so, the pylon points that belong to a given span and vegetation that can harm the power lines are extracted and this considerably reduces the point cloud size thus makes the subsequent process more efficient.

Figure 3 (b) shows the vertical corridor points $c_{p}$ close to one of the spans in the given segment. As shown in Figure 3 (c) these points are again converted into a binary image and a CCA is carried out to obtain the individual vegetation and pylons (see Figure $3(\mathrm{~d})$ ).

Assuming the maximum length or width of a pylon is $15 \mathrm{~m}$ and $10 \mathrm{~m}$, respectively, an area threshold $T_{a}=150 \mathrm{~m}^{2}$ is applied to remove trees with large horizontal areas. Ideally, trees with large horizontal areas can be extracted as above.

However, exceptions to this can happen when the bushes beneath the power lines is high and $c_{p}$ points make up the CCs including some pylons in them. In this situation, the component with area larger than $150 \mathrm{~m}^{2}$ (see Figure 3 (d)) are further slit into small components of size $150 \mathrm{~m}^{2}$ each. There should be no component exist with area greater than the given limit which is $150 \mathrm{~m}^{2}$, although in some cases two sub components can have points from same pylon. To validate this condition, the distance between extracted pylon points is estimated. If distance is less than a specified threshold, i.e., 10 $\mathrm{m}$ which is the maximum width of any pylon. It is assumed that points belong to the same pylon, otherwise they belong to different pylons. Due to the segmentation of dataset into subsets, only one pylon is usually encountered in a given span. As the minimum length of a span is $400 \mathrm{~m}$, and the segment may not contain the complete span from corridors.

At this stage, we will have isolated components containing

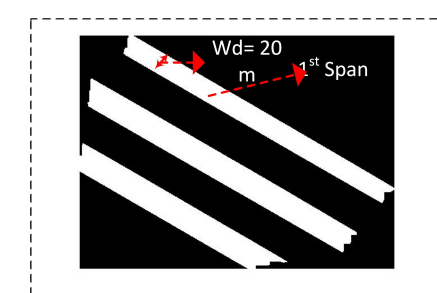

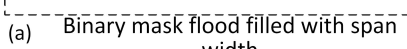
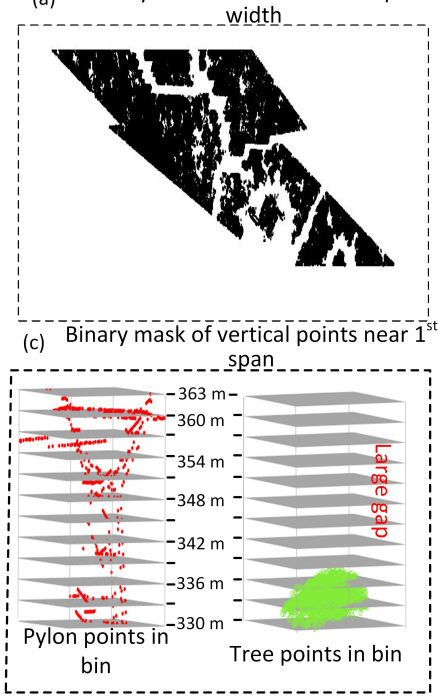

(e) Cuboid on individual connected Components in mask

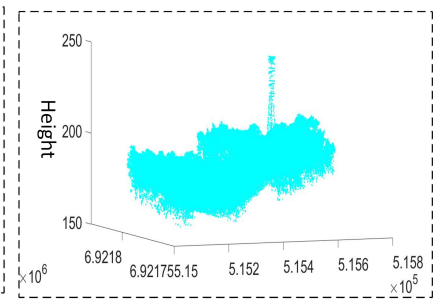

(b) Vertical object points near $1^{\text {st }}$ span
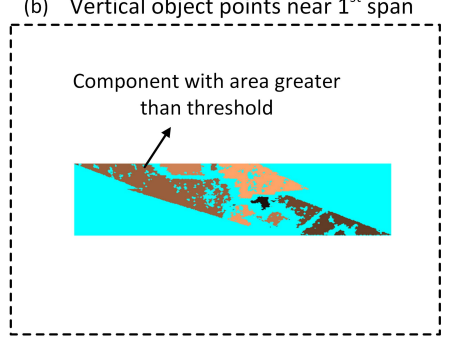

(d) Connected component analysis

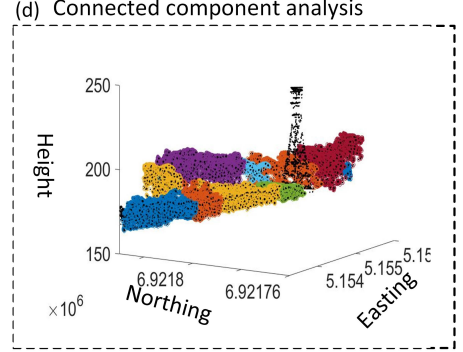

(f) Individual vegetation using size and area threshold
Fig. 3. Pylon and vegetation extraction in the a sample scene.

pylons or vegetation. In order to locate individual pylons and vegetation, the points $P_{b}$ in each $\mathrm{CC}$ or sub-component is individually investigated. The $3 \mathrm{D}$ cuboid is formed based on the minimum and maximum Easting $(x)$, Northing $(y)$ and Height $(z)$ values, where $x$ and $y$ values are estimated from $P_{b}$, but $z$ values is calculated from the maximum value of height of the wire points in a given span and the minimum height from the given $P_{b}$. These values are based on the fact that pylons reach the maximum heights of the power lines attached to them. Given that, the segment of the power lines nearest to the pylons are at a greater height than the segments of the power lines further away from the pylon. While the vegetation may reach the height of wires hung between the pylons and due to the sag in power lines which is maximum in the middle parts of wires, power lines may touch the tall vegetation beneath the span (see Figure 4).

Therefore, for a vegetation there should exists gap towards the top of the cuboid, but for a pylon there is no, or a little gap exists at the top (see Figure 3 (e)). The cuboid is then divided into bins $b_{n}$, where $n \geq 1$, the size of each $b_{n}$ is empirically set to be 1 . The number of points in each bin is counted, if there is at least 1 or more empty bin exists at the top for a component, that component is marked as a tree, otherwise as a pylon. Figure 3 (f) shows the vegetation in different colors and pylon points in black colour. Finally, for each pylon its location is obtained as the mean of its points. It is worth noting 
here, trees whose height is greater than pylons can be falsely extracted as pylons but in power line corridors the tall tree species are removed before the construction of PLC. So, the trees with larger height than the pylons do not exist in PLCs. However, the bushes beneath and around the vegetation can be grown up quickly and reach to the minimum height of wire and need to be monitored.

\section{Vegetation Monitoring}

As discussed earlier, the vegetation beneath and around the PLC needs to be monitored regularly. For the construction of PLC it is mandatory to clear the vegetation beforehand, so that PLC does not have trees under the span with height higher than pylons. However, the shrubs beneath and around the vegetation can grow up quickly and reach the minimum height of the wire. In countries like Australia, due to rain the growth of vegetation is rather fast. Moreover, in a hilly terrain vegetation with even small height can reach the height of power lines due to different height of terrain as shown in Figure 4.

Firstly, it is important to find out the required clearance

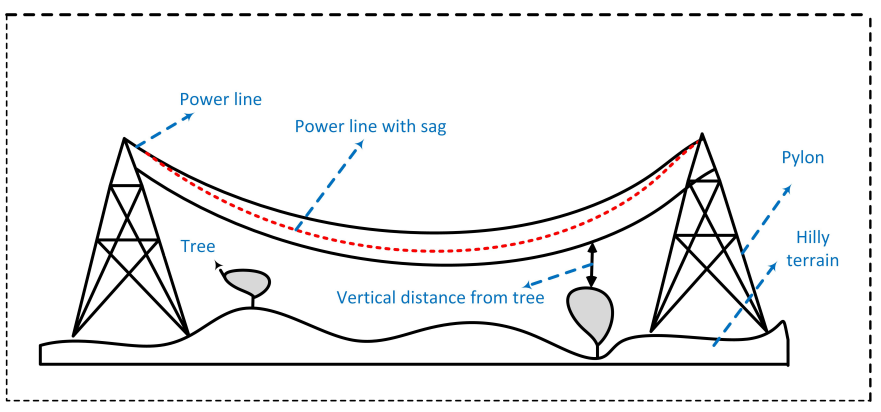

Fig. 4. Side view of a transmission line.

detachment of vegetation from power lines. We have defined two zones, i.e., clearance zone and danger zones for vegetation based on their location and vertical distance from the power lines as shown in Figure 4. The danger zone is defined as the location of the vegetation that could interfere with the power lines; and the clearance zone is the safe vegetation close to power lines at all times. For vegetation monitoring, the horizontal and vertical clearance in current legislation in Australia states that vegetation must be a minimum of $6.75 \mathrm{~m}$ from the lowest point of wires to allow for sag and sway of the power lines [20].

Instead of finding the horizontal distance of vegetation from the span, we assume that vegetation extracted in the previous step is close to power lines. This vegetation is located near to each span within $30 \mathrm{~m}$, i.e., $20 \mathrm{~m}$ width of span and $5 \mathrm{~m}$ on each side from outside the span. Thus, these locations are used further for determining the trees which can harm the power lines. To achieve this task, the height of each tree or vegetation is calculated with reference to minimum height of power line points (see Figure 4) if it is less than the threshold, it is considered to be in the danger zone. To identify the vegetation harmful to power lines, the vertical distance threshold i.e.,
$V_{d}=6 \mathrm{~m}$ is adopted from Electricity regulations Australia (www.legislation.vic.gov.au).

\section{PARAmeter Setting}

The list of parameters used for extraction of pylons and vegetation and for potential hazard analysis are set as shown in Table I . All of these parameters were first empirically set and tested for Bindebango (BDB) dataset and, then, without changing they were employed for Maindample (MDP) dataset (see Figure 5 for these datasets) for autonomous performance evaluation.

TABLE I

PARAMETERS SETTINGS

\begin{tabular}{llc}
\hline \multicolumn{1}{c}{ Parameters } & \multicolumn{1}{c}{ Values } & Sources \\
\hline Ground removal height & $1 \mathrm{~m}$ & {$[21]$} \\
Voxel size & $125 \mathrm{~m}^{3}$ & {$[9]$} \\
Mask resolution & $0.25 \mathrm{~m}$ & {$[21]$} \\
Span Width & $20 \mathrm{~m}$ & This paper \\
Corridor Width & $30 \mathrm{~m}$ & This paper \\
Pylon Width & $10 \mathrm{~m}$ & This paper \\
Pylon Height & $15 \mathrm{~m}$ & This paper \\
Horizontal Distance & $5 \mathrm{~m}$ & (www.legislation.vic.gov.au) \\
Vertical Distance & $6 \mathrm{~m}$ & (www.legislation.vic.gov.au) \\
\hline \hline
\end{tabular}

\section{PERformanCE Study}

In this section, the datasets, the ground truth data, results and discussion are outlined in detail.

\section{A. Datasets}

To test the feasibility of the proposed method, two datasets depicted in Figures 5 (a) and (b) are used from Maindample (MDP), Victoria and Bindebango (BDB), Queensland Australia with point density of 23.7 and 56.4 points per $\mathrm{m}^{2}$, respectively. Table II summarises their properties. Both sites have three transmission line corridors (TLCs).

The length of MDP is about twice the length of BDB, hence the points cloud of BDB increased proportionally. The width of MDP remain constant throughout the dataset at $330 \mathrm{~m}$ as shown in Figure 5 (a), while the width of BDB changes from $330 \mathrm{~m}$ and $530 \mathrm{~m}$. The third TLC of MDP is only $310 \mathrm{~m}$ in length and beneath the other two TLCs. It has only two pylons while there are 26 pylons in the other two TLCS in MDP. BDB has a total of 24 pylons from all three corridors. The MDP dataset is located on a flat area, and the BDB dataset is from a hilly area with dense vegetation underneath the wires, especially in TLC 1 of BDB (see Figure 5 (b)).

\section{B. Ground Truth}

Due to unavailability of the ground truth data a 3D interface was developed using MATLAB programming to manually collect the ground truth points. Table III shows the summary of the ground truth used for evaluation of results. As the size of each test dataset is very big so it is hard to collect ground truth for all points in the scene for evaluation.

Only the first six spans of one TLC from MDP site, and two spans from each TLC in BDB are used for the evaluation of 
TABLE II

SUMMARY OF TWO DATASETS

\begin{tabular}{ccccccc}
\hline Datasets & $\begin{array}{c}\text { Area } \\
\text { m }\end{array}$ & \multicolumn{3}{c}{ Points $\left(\times 10^{6}\right)$} & \multicolumn{3}{c}{ Number of pylons } \\
\cline { 3 - 7 } & $\mathbf{m}^{2}$ & All & Non-Ground & TLC 1 & TLC 2 & TLC 3 \\
\hline MDP & $5560 \times 330$ & 32.7 & 2.1 & 13 & 13 & 2 \\
BDP & $2500 \times 530$ & 18.5 & 14.5 & 8 & 8 & 8 \\
\hline
\end{tabular}

TABLE III

SUMMARY OF THE TWO GROUND TRUTH SITES.

\begin{tabular}{ccccccc}
\hline Site & $\begin{array}{c}\text { Area } \\
\mathbf{m}^{2}\end{array}$ & All & Pylon & Trees & Span & Pylons \\
\cline { 3 - 7 } & ${ }^{n}$ & 56,515 & 5,001 & 835 & 6 & 6 \\
\hline MDP & $1,170 \times 330$ & 435,579 & 3,860 & 395,657 & 6 & 6 \\
BDB & $667 \times 530$ & $\mathbf{4 9 2 , 0 9 4}$ & $\mathbf{8 , 8 6 1}$ & $\mathbf{3 9 6 , 4 9 2}$ & $\mathbf{1 2}$ & $\mathbf{1 2}$ \\
\hline Total & $\mathbf{1 8 3 7} \times \mathbf{8 6 0}$ & $\mathbf{4 9 2}$ & & &
\end{tabular}
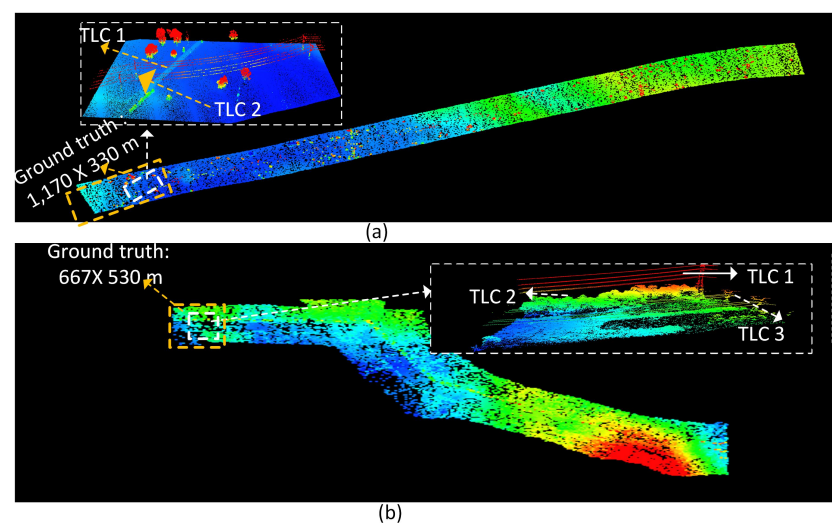

Fig. 5. The test datasets: (a) Maindample, Victoria; and (b) Bindebango, Queensland.

the proposed scheme. Points with height less than $1 \mathrm{~m}$ are not included in the ground truth data, as these points are assumed as terrain points.

\section{Evaluation Metrics}

For performance evaluation, object-based and point-based completeness $C_{m}$, correctness $C_{r}$ and quality $Q_{l}$ metrics are used [22].

For object-based evaluation, the total number of detected pylons is considered. In addition, for pylons, a localisation error $E_{l}$ (in both 2D, i.e., planimetric and 3D) is calculated as the distance between the mean points of an extracted pylon and its corresponding mean in the ground truth data.

\section{Results and Discussions}

Figure 6 displays the extracted pylons in two sites. Table IV shows the object-based completeness, correctness and quality values for the pylons in the two datasets. The two poles are missed from the MDP site and wrongly classified as vegetation. Thus, the object-based completeness and correctness of the MDP site are lower than those of the
BDB site. All other pylons (26 in MDP and 24 in BDB) were correctly extracted. In both sites no tree or vegetation was extracted as pylons. The localisation error $E_{l}$ of pylons in the BDB site is higher than that in the MDP site. Due to dense vegetation around pylons in the BDB site, bushes which are very close to pylons are extracted as pylon points (see Figure 5 (a)).

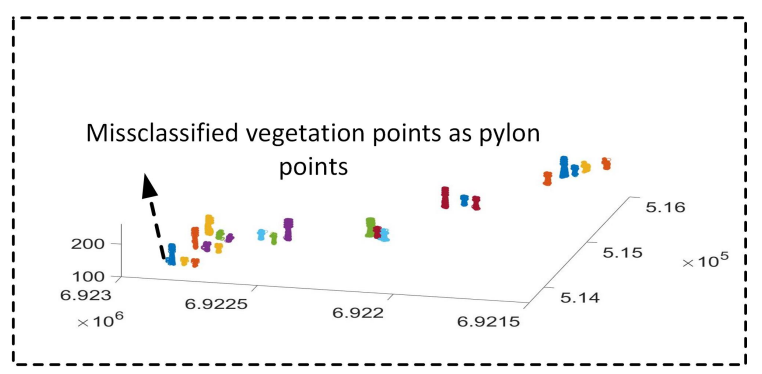

(a) Individual pylons from Bindebango (BDB) site

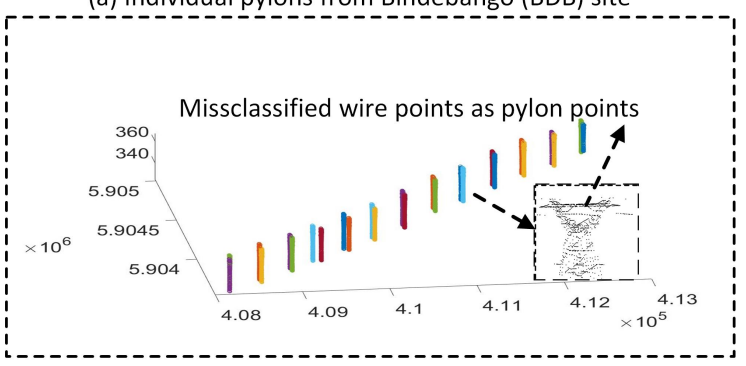

(b) Individual pylons from Maindample (MDP) site

Fig. 6. Individual pylon extraction.

Object-based evaluation results are not provided for vegetation. As the vegetation mostly exist in the form of shrubs near the PLC specifically in the BDB site, it is hard to count the individual trees or shrubs for object-based evaluation.

Table $\mathrm{V}$ shows the point-based evaluation results against the ground truth presented in Table III. In both datasets some of 
TABLE IV

OBJECT-BASED EVALUATION ON WHOLE DATASETS (ALL VALUES IN PERCENTAGE).

\begin{tabular}{cccc}
\hline Datasets & Pylons & $\begin{array}{c}\text { Pylo } \\
\text { Corr. }\end{array}$ & Qual. \\
\hline MDP & 98.3 & 98.5 & 97 \\
BDB & 100 & 100 & 100 \\
Average & $\mathbf{9 9 . 1}$ & $\mathbf{9 9 . 2}$ & $\mathbf{9 8 . 1}$ \\
\hline
\end{tabular}

the power line points are extracted as pylon points, as they are very close to the pylons and it is hard to separate them.

Also, due to dense vegetation around the pylons in the BDB site, some of the tree points are extracted as pylon points (see Figure 6 (a)) resulting in low correctness and completeness. For trees, we only considered the vegetation that exists within $30 \mathrm{~m}$ from the corridor, the rest of the vegetation points are not considered. Consequently, completeness and correctness appear low. In addition, some of the pylon points are classified as vegetation points near the pylon.

Figure 7 shows the extracted vegetation and potential hazard analysis in PLC. Vegetation in the form of big bushes are divided into small segments, where each segment contains bushes with small area (see Figure 7 (e) and (g)). The trees in red in Figure 7 (b) show they are at potential risk and can be dangerous to power lines. Hence, they are in the danger zone and need to be cleared. While the rest of the trees shown in green are in the safe zone.

\section{CONCLUSION}

This paper presents a method to extract individual pylons and vegetation in PLC and provides an explicit framework to detect the trees and bushes that can harm the power lines. The proposed method uses unsupervised techniques to find the isolated pylons and trees. Also, the vegetation which exists in the form of bushes is divided into small segments to find the potential threats from them.

To our knowledge, this is the first method that employs ALS to identify the potential hazards from vegetation in PLC in a complex environment. Most of the existing methods used MLS and aerial images to detect trees to prevent the risk from tall trees growing closely to power lines. The proposed method is tested on two different large datasets with flat and hilly terrain with comparatively low point density and shows $96.5 \%$ and $100 \%$ completeness for pylons and vegetation. However, the method does not detect buildings and other objects which make its application less feasible in urban areas. Also in urban areas where the poles and trees are similar additional steps (post-processing) will be required to separate them. The proposed method is rather general for complex datasets that has high voltage power lines in forest area with dense vegetation and mountainous terrain. In future, the modelling of power lines and pylons will be investigated which is very important for automated modelling of PLC.

\section{REFERENCES}

[1] L. Matikainen, M. Lehtomäki, E. Ahokas, J. Hyyppä, M. Karjalainen, A. Jaakkola, A. Kukko, and T. Heinonen, "Remote sensing methods for power line corridor surveys," ISPRS Journal of Photogrammetry and Remote Sensing, vol. 119, pp. 10-31, 2016.
[2] I. Ituen and G. Sohn, "The way forward: Advances in maintaining rightof-way of transmission lines," Geomatica, vol. 64, no. 4, pp. 451-462, 2010.

[3] R. Aggarwal, A. Johns, J. Jayasinghe, and W. Su, "An overview of the condition monitoring of overhead lines," Electric Power systems research, vol. 53, no. 1, pp. 15-22, 2000.

[4] X. Qin, G. Wu, J. Lei, F. Fan, and X. Ye, "Detecting inspection objects of power line from cable inspection robot lidar data," Sensors, vol. 18 , no. 4, p. 1284,2018

[5] S. J. Mills, M. P. G. Castro, Z. Li, J. Cai, R. Hayward, L. Mejias, and R. A. Walker, "Evaluation of aerial remote sensing techniques for vegetation management in power-line corridors," IEEE Transactions on Geoscience and Remote Sensing, vol. 48, no. 9, pp. 3379-3390, 2010.

[6] K. Sarabandi and M. Park, "Extraction of power line maps from millimeter-wave polarimetric sar images," IEEE Transactions on Antennas and Propagation, vol. 48, no. 12, pp. 1802-1809, 2000.

[7] G. R. Stockton and A. Tache, "Advances in applications for aerial infrared thermography," in Thermosense XXVIII, vol. 6205, p. 62050C, International Society for Optics and Photonics, 2006.

[8] J. Yang and Z. Kang, "Voxel-based extraction of transmission lines from airborne lidar point cloud data," IEEE Journal of Selected Topics in Applied Earth Observations and Remote Sensing, vol. 11, no. 10, pp. 3892-3904, 2018.

[9] H. Kim and G. Sohn, "3d classification of power-line scene from airborne laser scanning data using random forests," Int. Arch. Photogramm. Remote Sens, vol. 38, pp. 126-132, 2010.

[10] B. Guo, X. Huang, F. Zhang, and G. Sohn, "Classification of airborne laser scanning data using jointboost," ISPRS Journal of Photogrammetry and Remote Sensing, vol. 100, pp. 71-83, 2015.

[11] G. Sohn, Y. Jwa, and H. B. Kim, "Automatic powerline scene classification and reconstruction using airborne lidar data," ISPRS Ann. Photogramm. Remote Sens. Spat. Inf. Sci, vol. 13, no. 167172, p. 28 2012.

[12] M. Awrangjeb, D. Jonas, and J. Zhou, "An automatic technique for power line pylon detection from point cloud data," in 2017 International Conference on Digital Image Computing: Techniques and Applications (DICTA), pp. 1-8, IEEE, 2017.

[13] M. Awrangjeb, "Extraction of power line pylons and wires using airborne lidar data at different height levels," Remote Sensing, vol. 11, no. 15 , p. $1798,2019$.

[14] L. Ma and Y. Chen, "Aerial surveillance system for overhead power line inspection," Center for Self-Organizing and Intelligent Systems (CSOIS), Utah State Univ., Logan, Tech. Rep. USU-CSOIS-TR-04-08 (September 2000), 2004.

[15] C. Sun, R. Jones, H. Talbot, X. Wu, K. Cheong, R. Beare, M. Buckley, and $\mathrm{M}$. Berman, "Measuring the distance of vegetation from powerlines using stereo vision," ISPRS journal of photogrammetry and remote sensing, vol. 60, no. 4, pp. 269-283, 2006.

[16] A. Kukko, H. Kaartinen, J. Hyyppä, and Y. Chen, "Multiplatform mobile laser scanning: Usability and performance," Sensors, vol. 12, no. 9, pp. 11712-11733, 2012.

[17] Z. Shi, Y. Lin, and H. Li, "Extraction of urban power lines and potential hazard analysis from mobile laser scanning point clouds," International Journal of Remote Sensing, vol. 41, no. 9, pp. 3411-3428, 2020.

[18] Q. Chen, D. Baldocchi, P. Gong, and M. Kelly, "Isolating individual trees in a savanna woodland using small footprint lidar data," Photogrammetric Engineering \& Remote Sensing, vol. 72, no. 8, pp. 923-932, 2006.

[19] Z. Li, T. S. Bruggemann, J. J. Ford, L. Mejias, and Y. Liu, "Toward automated power line corridor monitoring using advanced aircraft control and multisource feature fusion," Journal of Field Robotics, vol. 29, no. 1, pp. 4-24, 2012.

[20] D. J. Clarke, K. A. Pearce, and J. G. White, "Powerline corridors: degraded ecosystems or wildlife havens?," Wildlife Research, vol. 33, no. 8, pp. 615-626, 2007.

[21] N. Munir, M. Awrangjeb, B. Stantic, G. Lu, and S. Islam, "Voxel-based extraction of individual pylons and wires from lidar point cloud data," ISPRS Annals, 2019.

[22] Y. Wang, Q. Chen, L. Liu, D. Zheng, C. Li, and K. Li, "Supervised classification of power lines from airborne lidar data in urban areas," Remote Sensing, vol. 9, no. 8, p. 771, 2017. 
TABLE V

Point-BASED EVALUATION ON WHOLE DATASETS (ALL VALUES IN PERCENTAGE).

\begin{tabular}{ccccccccc}
\hline Datasets & & \multicolumn{3}{c}{ Pylons } & \multicolumn{3}{c}{ Trees } \\
\cline { 3 - 9 } & Comp. & Corr. & Qual. & 2D error. & 3D error. & Comp. & Corr. & Qual. \\
\hline MDP & 98 & 100 & 99.2 & 0.12 & 0.31 & 100 & 90 & 91.2 \\
BDB & 95 & 97 & 91 & 0.13 & 0.61 & 100 & 71.7 & 70.1 \\
Average & $\mathbf{9 6 . 5}$ & $\mathbf{9 8 . 5}$ & $\mathbf{9 5 . 1}$ & $\mathbf{0 . 1 2}$ & $\mathbf{0 . 4 6}$ & $\mathbf{1 0 0}$ & $\mathbf{8 0 . 8 5}$ & $\mathbf{8 0 . 6}$ \\
\hline
\end{tabular}
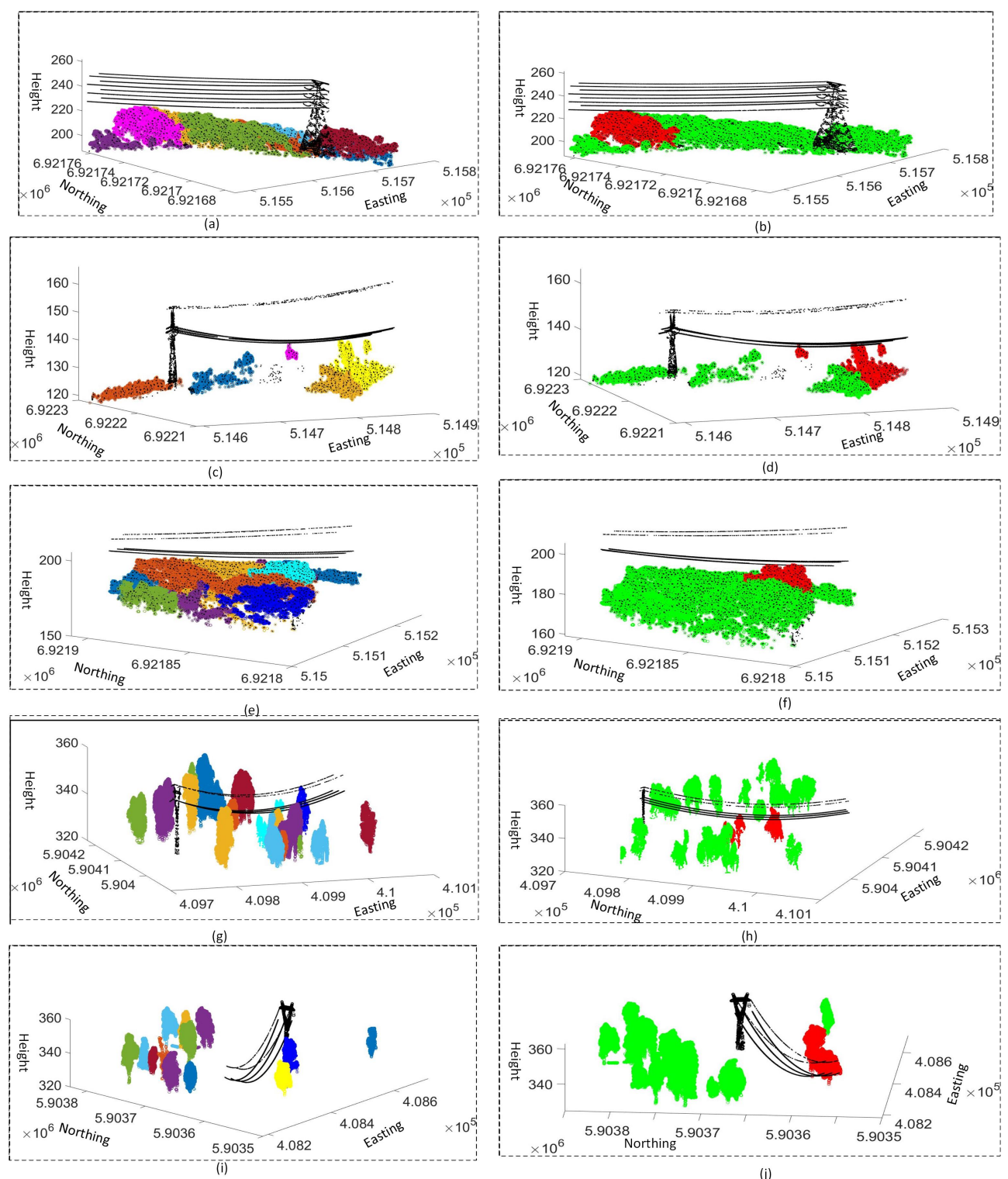

Fig. 7. Vegetation extraction and potential hazard analysis. 